\title{
High-energy x-ray diffraction from surfaces and nanoparticles
}

\author{
U. Hejral, ${ }^{1,2,3,}{ }^{*}$ P. Müller, ${ }^{1,2}$ M. Shipilin, ${ }^{3}$ J. Gustafson, ${ }^{3}$ D. Franz, ${ }^{1,2}$ R. Shayduk, ${ }^{1,4}$ U. Rütt, ${ }^{1,5}$ C. Zhang, ${ }^{3}$ L. R. Merte,,${ }^{3,6}$ \\ E. Lundgren, ${ }^{3}$ V. Vonk, ${ }^{1}$ and A. Stierle ${ }^{1,2, \dagger}$ \\ ${ }^{1}$ Deutsches Elektronen-Synchrotron (DESY), D-22603 Hamburg, Germany \\ ${ }^{2}$ Fachbereich Physik Universität Hamburg, Jungiusstrasse 9, D-20355 Hamburg, Germany \\ ${ }^{3}$ Synchrotron Radiation Research, Lund University, Box 118, SE-221 00 Lund, Sweden \\ ${ }^{4}$ European XFEL GmbH, Holzkoppel 4, D-22869 Schenefeld, Germany \\ ${ }^{5}$ Argonne National Laboratory, Lemont, Illinois 60439, USA \\ ${ }^{6}$ Max IV Laboratory, Fotongatan 8, SE-22594 Lund, Sweden
}

(Received 3 August 2017; revised manuscript received 27 October 2017; published 27 November 2017)

\begin{abstract}
High-energy surface-sensitive $\mathrm{x}$-ray diffraction (HESXRD) is a powerful high-energy photon technique $(E>70 \mathrm{keV})$ that has in recent years proven to allow a fast data acquisition for the 3D structure determination of surfaces and nanoparticles under in situ and operando conditions. The use of a large-area detector facilitates the direct collection of nearly distortion-free diffraction patterns over a wide $q$ range, including crystal truncation rods perpendicular to the surface and large-area reciprocal space maps from epitaxial nanoparticles, which is not possible in the conventional low-photon energy approach $(E=10-20 \mathrm{keV})$. Here, we present a comprehensive mathematical approach, explaining the working principle of HESXRD for both single-crystal surfaces and epitaxial nanostructures on single-crystal supports. The angular calculations used in conventional crystal truncation rod measurements at low-photon energies are adopted for the high-photon-energy regime, illustrating why and to which extent large reciprocal-space areas can be probed in stationary geometry with fixed sample rotation. We discuss how imperfections such as mosaicity and finite domain size aid in sampling a substantial part of reciprocal space without the need of rotating the sample. An exact account is given of the area probed in reciprocal space using such a stationary mode, which is essential for in situ or operando time-resolved experiments on surfaces and nanostructures.
\end{abstract}

DOI: 10.1103/PhysRevB.96.195433

\section{INTRODUCTION}

Surface-sensitive x-ray diffraction (SXRD) allows a quantitative determination of the atomic structure of truncated realspace objects with internal periodicity, such as single crystals or crystalline faceted nanoparticles, exhibiting well-defined surfaces and interfaces [1-7]. Because of the compatibility of $\mathrm{x}$-ray diffraction experiments with harsh sample environments, such as elevated temperatures, reactive gas mixtures at atmospheric pressures, or strong electromagnetic fields, in the last decade SXRD has opened unique opportunities for the investigation of surfaces and nanostructures under application relevant conditions [8-15]. In the case of single crystals, the surface structures are retrieved from the analysis of intensity variations along sets of crystal truncation rods (CTRs), which are lines in reciprocal space of nonzero diffracted intensity oriented perpendicular to the respective surface and interconnecting the corresponding Bragg peaks of the bulk material. To measure the integrated intensities at various points along the CTRs, the sample needs to be rotated in the traditional SXRD rocking-scan mode ( $E=10-20 \mathrm{keV})$ around its surface normal at each point ("rocking scan" [4]), which is, however, rather time-consuming (on the order of hours or days for a complete set). For epitaxial nanoparticles supported by single-crystalline substrates, extended reciprocal-space maps need to be recorded, which can be simulated to retrieve the average nanoparticle shape and size [11-13]. They also involve time-consuming sample and detector movements.

\footnotetext{
*uta.hejral@sljus.lu.se

†andreas.stierle@desy.de
}

High-energy surface $\mathrm{x}$-ray diffraction (HESXRD) is a novel tool to investigate the atomic structure of surfaces, interfaces, and ensembles of nanoparticles [16-20], which became feasible with the advent of intense, microfocused hard $\mathrm{x}$-ray beams of high-photon energy $(E>70 \mathrm{keV})$ at third-generation synchrotron radiation sources [21]. In combination with a large two-dimensional detector, it allows, without scanning the detector, for an immediate and nearly distortion-free mapping of vertical planes in reciprocal space, also containing, among others, CTR and nanoparticle facet diffraction signals. Accordingly, HESXRD provides, with subsecond temporal resolution, atomic-scale structural information without the need for detector movement or extended sample scanning. This is essential for the in situ and operando investigation of kinetic processes on single-crystal surfaces, interfaces, and epitaxial nanostructures. In addition, the performance of rocking scans while taking 2D images allows a very fast high-resolution mapping of reciprocal space for single-crystal surfaces (on the order of $10 \mathrm{~min}$. [16,17]).

This results in a 3D dataset of integrated intensities of CTR diffraction signals, which is not feasible on comparable time scales at conventional photon energies. This turns out to be crucial during the search for unknown or unexpected structures, which may be metastable under reaction or processing conditions. However, a reciprocal-space parametrization in terms of the diffraction angles at high-photon energies and a thorough discussion of the role of sample imperfections on the diffraction signal are lacking so far.

Here we shed light on the working principles of HESXRD: in the first section we will demonstrate how the angular calculations for the scattering angles from Ref. [2], valid for 
the case of conventional photon energies used in SXRD, can be approximated for the case of high-photon energies and small diffraction angles. The result will illustrate why, from a mathematical point of view, a nearly distortion-free mapping of vertical planes in reciprocal space is possible for a relevant range of momentum transfers. In the second section, these equations will be applied to the case of samples featuring nanostructured objects (nanoparticles, or domains inside a single crystal) with small domain sizes on the order of $10 \mathrm{~nm}$ and an angular distribution of these domains around a preferred direction ("in-plane mosaicity"). We will show to what extent such intrinsic sample imperfections in combination with highphoton energies result in an enlargement of the detectable reciprocal-space area in stationary geometry, which allows for a 2D mapping without the need to rotate the sample, enabling fast, time-resolved in situ or operando studies on surfaces and nanostructures.

\section{APPROXIMATION OF CRYSTAL TRUNCATION ROD DIFFRACTION-ANGLE CALCULATIONS FOR HIGH-PHOTON ENERGIES}

An x-ray beam impinging onto the sample surface results in a diffraction pattern in reciprocal space, in which the real-space positions of the atoms determine the positions and intensities of the diffracted signals to be probed by the detector. In the following, the calculations for these diffraction angles and accordingly required detector positions will be conveyed from the conventional ( $E=10-20 \mathrm{keV}, \mathrm{SXRD})$ to the high-photon-energy regime ( $E>70 \mathrm{keV}$, HESXRD).

Figure 1(a) illustrates the principal diffraction geometry for the case of conventional photon energies (SXRD). An X-ray beam of distinct photon energy $E$ and wavelength $\lambda=\frac{h c}{E}$ impinges under a grazing angle $\alpha$ onto the sample surface. Its incident wave vector $\overrightarrow{k_{\mathrm{i}}}$ with magnitude $k=\frac{2 \pi}{\lambda}=\frac{E}{\hbar \mathrm{c}}$ defines the radius of the Ewald sphere on which the observable reciprocal space lies.

To measure the diffracted intensity at a distinct reciprocal lattice point $\vec{G}=h \overrightarrow{a^{*}}+k \overrightarrow{b^{*}}+1 \overrightarrow{c^{*}}$ defined by the sample's reciprocal lattice vectors $\overrightarrow{a^{*}}, \overrightarrow{b^{*}}, \overrightarrow{c^{*}}$, the diffraction condition

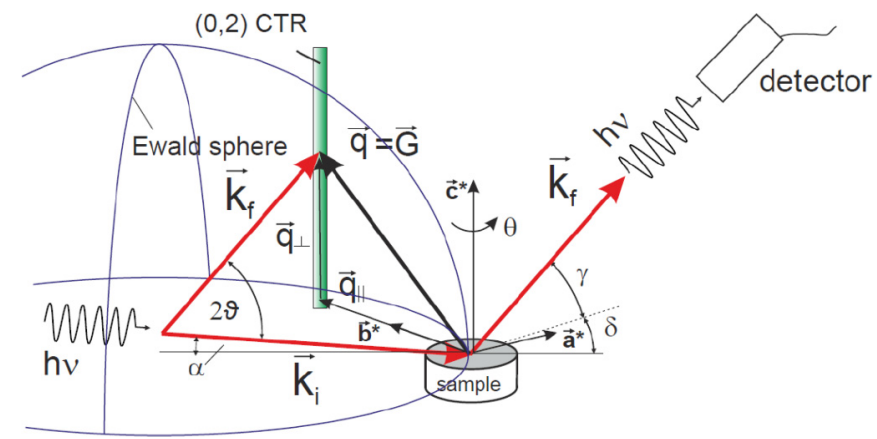

FIG. 1. Surface x-ray diffraction geometry at conventional photon energies in the $10-20-\mathrm{keV}$ regime illustrating the scattering geometry and angles introduced in the text for a vertical $(2+2)$ diffractometer with the detector rotations $\delta$ and $\gamma$ independent of the sample rotations $\theta$ and $\alpha$, similar to the geometry for a stationary $2 \mathrm{D}$ detector [22].
$\vec{G}=\vec{q}$ needs to be fulfilled, in which the total momentum transfer $\vec{q}=\overrightarrow{k_{\mathrm{f}}}-\overrightarrow{k_{\mathrm{i}}}\left(q=\frac{4 \pi}{\lambda} \sin \vartheta\right.$, with total diffraction angle $2 \vartheta$ ) is determined by the respective final and incident wave vectors $\overrightarrow{k_{\mathrm{f}}}$ and $\overrightarrow{k_{\mathrm{i}}}$ with magnitude $k=k_{\mathrm{f}}=k_{\mathrm{i}}$. Accordingly, a certain reciprocal-space lattice point is probed only if its position coincides with the Ewald sphere and if the detector is positioned along the $k_{\mathrm{f}}$ direction. As an example, Fig. 1 shows the scenario where the diffracted intensity is measured at a point on the sample's $(0,2,1)$ rod, which accordingly corresponds to the intersection point of the rod with the Ewald sphere.

To collect crystal truncation rod data, as well as extended reciprocal space maps, the in- and out-of-plane diffraction angles $\delta$ and $\gamma$ in the laboratory frame, both realized by the detector arm, as well as the sample rotation $\theta$ around the surface normal, need to be varied when using lowphoton energies. A convenient parametrization of the intrinsic diffraction angles with respect to the angles of the sample surface $\gamma^{\prime}$ and $\delta^{\prime}$ is given by [2]

$$
\begin{gathered}
\sin ^{2} \gamma^{\prime}=\sin ^{2} \alpha+4 \cos ^{2} \alpha \sin \theta_{\mathrm{B}}\left(\sin \theta-\sin \theta_{\mathrm{B}}\right), \\
\sin \delta^{\prime}=\frac{2 \cos \theta \sin \theta_{\mathrm{B}}}{\left[1-4 \sin \theta_{\mathrm{B}}\left(\sin \theta-\sin \theta_{\mathrm{B}}\right)\right]^{1 / 2}}, \\
\cos 2 \vartheta=\cos \delta \cos \gamma .
\end{gathered}
$$

Therein, Eq. (1) describes how the exit angle $\gamma^{\prime}$ with respect to the surface plane is connected to the sample rotation $\theta$ when following a CTR perpendicular to the surface (incident angle $\alpha$ fixed). In Eq. (2) the corresponding tracking of the diffraction angle $\delta^{\prime}$ in the surface plane is given. Both equations contain the in-plane Bragg angle $\theta_{\mathrm{B}}$, which is defined by $\vec{q}_{\|}=$ $2 k \sin \left(\theta_{\mathrm{B}}\right)$, where $\vec{q}_{\|}$corresponds to the parallel component of the momentum transfer $\vec{q}=\vec{q}_{\|}+\vec{q}_{\perp}$ in the limit of $\alpha \approx$ $0, \gamma \approx 0$. Equation (3) describes the total diffraction angle 29 , which is illustrated in Fig. 1 . In the limit of small-angle incident angles $(\alpha \approx 0)$, as considered here, it can be shown that $\gamma=\gamma^{\prime}$ and $\delta=\delta^{\prime}$ [22], and we will make use of these relations in the following.

To collect the data needed for the CTR analysis, two different approaches were established in the past using conventional photon energies. The first one is the traditional crystallography approach using a point detector with defined aperture combined with rocking scans at each reciprocal lattice point $\vec{G}$ in reciprocal space along the crystal truncation rods [4]. In the second approach, a two-dimensional detector is mounted on the detector arm and the intersection of the reciprocal lattice truncation or surface rods gives rise to a diffraction spot (for high $l$ values), which can be integrated after background subtraction [23]. At low $l$, rocking scans still need to be performed. Hence, either way, rocking scans at various points along a CTR cannot be avoided.

This changes when high-energy photons $(E>70 \mathrm{keV})$ as in HESXRD are employed. Figure 2 illustrates how, with increasing photon energy, the Ewald sphere flattens such that more structural information per detector area is probed within a single snapshot, allowing for a complete avoidance, or at least a significant reduction, of rocking scans. At the same time, the 
(a)
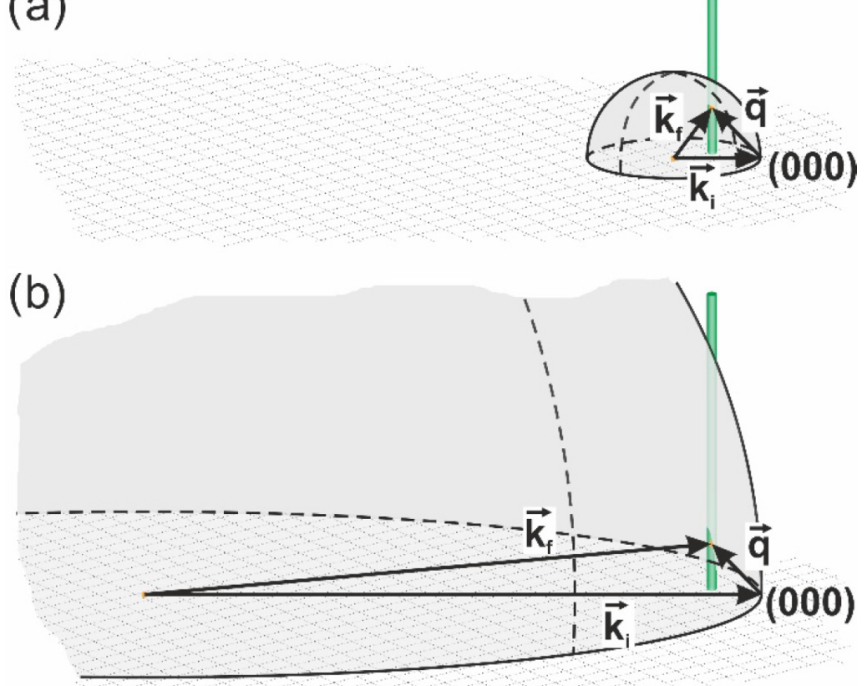

FIG. 2. Surface x-ray diffraction geometry at (a) conventional ( $E=12.4 \mathrm{keV})$ and (b) high-photon energies $(E=88.6 \mathrm{keV})$ drawn using the same scale.

scattering angles decrease significantly. As an example, the total scattering angle of the $\operatorname{Pd}(200)$ Bragg peak comprises $2 \vartheta_{1}=20.94^{\circ}$ at a low-photon energy of $E_{1}=12.4 \mathrm{keV}$, but only $2 \vartheta_{2}=2.92^{\circ}$ at a high-photon energy of $E_{2}=88.6 \mathrm{keV}$.

Accordingly, the use of high-photon energies makes a small-angle approximation feasible, which allows a reformulation of the aforementioned scattering angle parametrization (see Appendix):

$$
\begin{gathered}
\gamma \cong 2 \sqrt{\theta_{\mathrm{B}}\left(\theta-\theta_{\mathrm{B}}\right)}, \\
\delta \cong 2 \theta_{\mathrm{B}}, \\
2 \vartheta \cong \sqrt{\delta^{2}+\gamma^{2}} .
\end{gathered}
$$

The sets of equations for the parametrization of scattering angles at conventional [Eqs. (1)-(3)] and high-photon energies [Eqs. (1a)-(3a)] allow for a direct comparison of the diffracted beam trajectories along CTRs in angular space, which will be discussed in the following.

Figures 3(a) and 3(b) reveal various CTR trajectories for the wavelengths $\lambda=0.1 \mathrm{~nm}(E=12.4 \mathrm{keV}$, conventional regime $)$ and $\lambda=0.014 \mathrm{~nm}(E=88.6 \mathrm{keV}$, high-energy regime), respectively. The trajectories (black lines) are plotted as a function of the in- and out-of-plane scattering angles $\delta$ and $\gamma$ in the $(\delta, \gamma)$ plane of the detector. Note that both plots cover the same in-and out-of-plane momentum transfer range $q_{\|}$and $q_{\perp}$ of 70 and $60 \mathrm{~nm}^{-1}$, respectively. Along a CTR trajectory, the in-plane momentum transfer $q_{\|}$is constant and only $q_{\perp}$ is varied. At $\gamma=0$ (and accordingly $l=q_{\perp}=0$ ), the trajectory of a distinct CTR starts at a $\delta$ value corresponding to the (constant and energy-independent) in-plane momentum transfer $q_{\|}$. For $\lambda=0.1 \mathrm{~nm}(E=12.4 \mathrm{keV}$, in the conventional regime) the rod trajectories are curved and they level off at specific momentum transfers $q_{\perp}=\frac{2 \pi}{\lambda} \sin \gamma$ perpendicular (a)
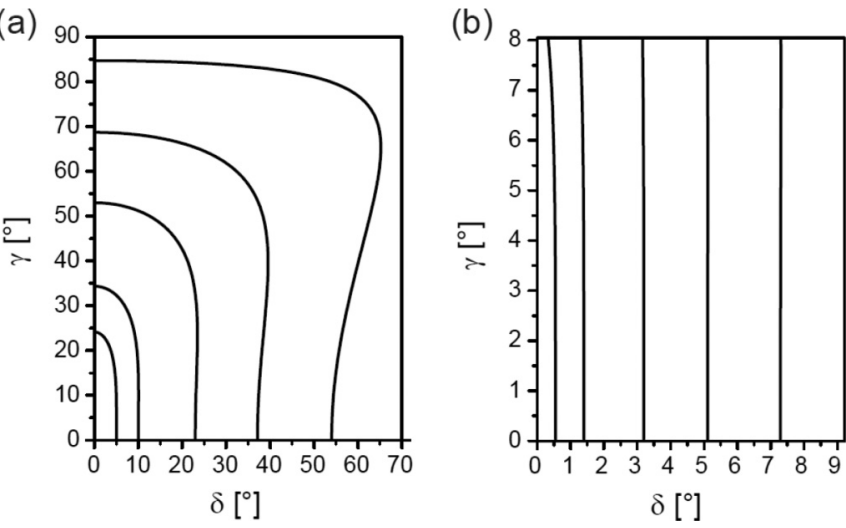

FIG. 3. Comparison of trajectories (black lines) of the diffracted beam along various CTRs as followed by the detector arm $[(\delta, \gamma)$ plane] at conventional (a) $12.4 \mathrm{keV}$ and high-photon energies (b) $88.6 \mathrm{keV}$, when the sample is rotated around its surface normal. The plots in (a) and (b) cover the same in- and out-of-plane momentum transfer range $q_{\|}$and $q_{\perp}$ of 70 and $60 \mathrm{~nm}^{-1}$, respectively.

to the surface, with further small increase requiring a large movement in $\theta$ limiting the range in accessible $q_{\perp}$.

Contrary, for the higher-photon energy $(\lambda=0.014 \mathrm{~nm})$, the rod trajectories in the $(\delta, \gamma)$ plane of the detector correspond to almost straight lines, in agreement with nearly constant values of $\delta$, as predicted by Eq. (2a), representing a direct image of crystal truncation rods in reciprocal space. The sample rotation $\theta$ varies only on the order of $2 \theta_{\mathrm{B}}$ (few degrees) when following a CTR over a range of $\vec{q}_{\perp}$ typically investigated in experiments. In small-angle approximation the reciprocal lattice index $l$ is directly proportional to the exit angle $\gamma$.

Further advantage of the high-energy beam trajectories can be seen in the possibility to measure a wide $\vec{q}_{\perp}$-range also for low in-plane momentum transfers, as they might occur for surface reconstructions. This stands in contrast to the lowphoton energy case, in which only a limited $\vec{q}_{\perp}$-range can be reached. Accordingly, the high-energy approach helps to overcome one of the shortcomings of traditional SXRD as compared to low-energy electron diffraction (LEED), which allows the measurement of high $\vec{q}_{\perp}$ values for small $\vec{q}_{\|}$values [24].

To illustrate the validity of Eq. (1a), Fig. 4 shows experimental data obtained from a $\operatorname{Pd}(001)$ single crystal investigated at high-photon energies $(E=85 \mathrm{keV})$. The sequence of images shown in Fig. 4(a) displays the diffracted beam on the 2D detector measured at selected $\theta$ values during a rocking scan of constant step size $\Delta \theta$ when scanning along the $\operatorname{Pd}(11 \mathrm{~L})$-CTR (fcc bulk coordinates). The last image in Fig 4(a) was obtained by superimposing all 75 detector images performed during the scan, where for each pixel the highest intensity in all of the detector frames was plotted. Accordingly, it corresponds to the detector $(\delta, \gamma)$ plane of Fig. 3(b), and confirms experimentally the almost straight trajectories of negligible variation in $\delta$ as predicted by Eq. (2a).

Figure 4(b) displays the experimental $\gamma$ values (circles) plotted as a function of the sample rotation $\theta$. They were deduced from the respective intersection points of the CTR with the Ewald sphere (positions of highest intensity) in 
(a)

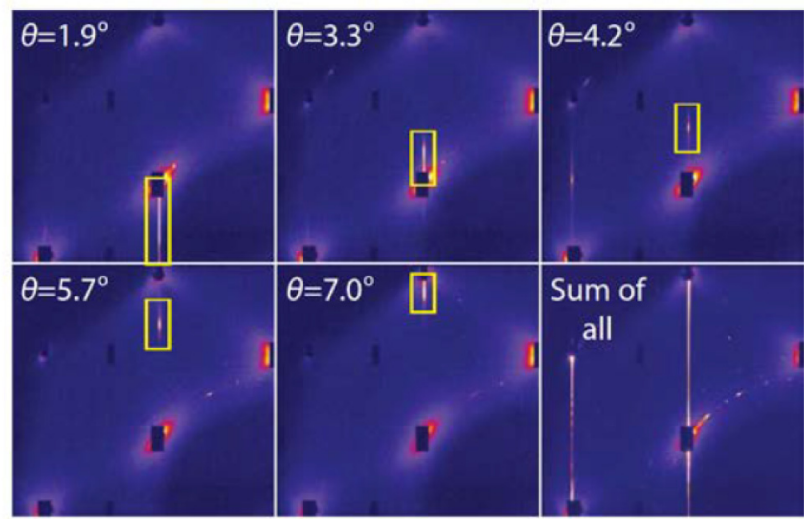

(b)

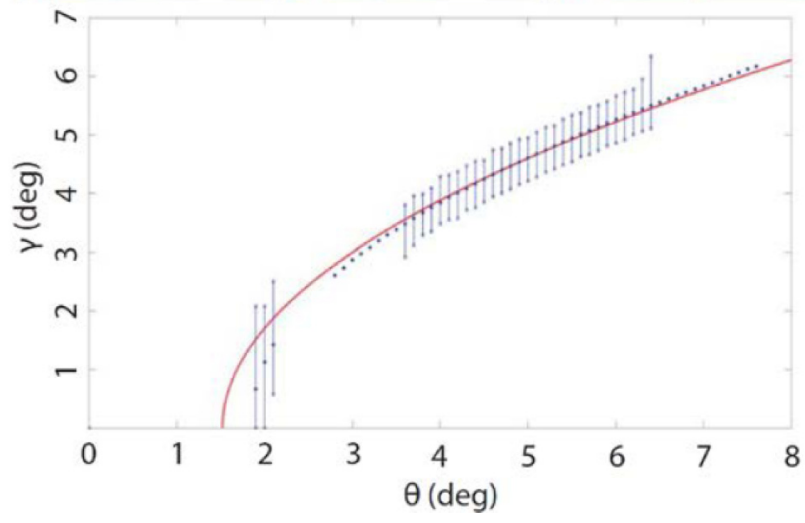

FIG. 4. (a) High-energy x-ray diffraction patterns from a $\operatorname{Pd}(100)$ surface at selected $\theta$ values taken during a rocking scan over a range of approximately $7^{\circ}$; last image: superposition of all 75 detector images performed during the scan plotting for each pixel the highest intensity in all of the detector frames. The boxes mark the intersection region of the reciprocal lattice with the Ewald sphere. (b) Experimental $\gamma$ values of the respective intersection points between the CTR and the Ewald sphere (circles) as a function of the sample rotation $\theta$; the red solid line represents the calculated dependency according to Eq. (1a); the blue bars correspond to the range $\Delta \gamma$ along the CTR within which still $25 \%$ of the maximum intensity of the intersection point was monitored. The blue bars are missing for the cases in which this intensity range was blocked by beamstops securing the $2 \mathrm{D}$ detector from the high-intensity Pd Bragg peaks.

the individual 2D maps as the ones shown in Fig. 4(a). The experimental data follow the square-root dependency predicted by the derived equation (1a) (red line), which is thus affirmed experimentally. For a measurement with equal step size $\Delta l$, the rotation step width can be adopted according to Eq. (1a).

As can be inferred from the 2D maps measured at a fixed $\theta$ value [Fig. 4(a)], not only the respective intersection points of the CTR with the Ewald sphere are detectable [circles in Fig. 4(b)]; in addition, there is also measurable signal above and below the respective intersection points. Their extent in area along the CTR decreases with increasing $\gamma$ values, as for low $\gamma$ values the angle between the CTR and the Ewald sphere decreases significantly. The respective intensity ranges are illustrated by the blue bars in Fig. 4(b), which correspond to the range $\Delta \gamma$ along the CTR within which still $25 \%$ of the maximum intensity at the intersection point was monitored. It will be discussed in the following that they result from the presence of finite-size crystallite domains featuring an angular distribution with respect to a preferential orientation (in-plane mosaicity). Due to their small mosaicity (around $0.05^{\circ}$ ) and the large surface domain size (typically 100-200 nm) of the investigated crystal, the diffracted beams for individual steps in the $\theta$ scan are still discernible along the Pd CTRs [see Fig. 4(a), sum of all images]. Note that the typical energy resolution $\frac{\Delta \mathrm{E}}{\mathrm{E}}=$ $\frac{\Delta \vartheta}{\vartheta} \approx 2 \times 10^{-3}$ in high-energy diffraction experiments corresponds to an angular peak broadening $\Delta \vartheta$ which is of the order of $5 \times 10^{-3^{\circ}}$ and plays a role for perfect single crystals only.

\section{STATIONARY DIFFRACTION GEOMETRY (FIXED SAMPLE ROTATION $\theta_{\mathrm{S}}$ ) FOR IN SITU STUDIES OF EPITAXIAL NANOSTRUCTURES}

So far we have considered a variable sample rotation $\theta$ to follow the beam trajectories discussed above. However, during operando studies or time-resolved experiments, processes are usually too fast to be followed by azimuthal sample (rocking) scans. Accordingly, probing a large section in reciprocal space at a fixed (stationary) sample rotation $\theta_{\mathrm{S}}$ is desirable. In the following we will elucidate why mosaic spreads of finite-size crystalline domains within samples are highly beneficial in this respect. In addition, the thus-gained detectable area in reciprocal space at a fixed sample rotation $\theta_{\mathrm{S}}$ will be estimated, which facilitates time-resolved operando studies by the sequential recording of $2 \mathrm{D}$ diffraction patterns.

In general, crystalline samples feature intrinsic imperfections. For metal single crystals, they include finite-size crystallite domains exhibiting angular distributions on the order of $0.05-0.1^{\circ}$ around a preferred direction. In the case of epitaxial nanoparticles on single-crystal oxide supports, it is the weak interaction between the metal particles and the oxide that triggers angular distributions of the nanoparticles, typically on the order of $1-3^{\circ}$. In this case effects of the typical energy broadening $\frac{\Delta E}{E}=2 \times 10^{-3}$ on the signal can be neglected (see Sec. II).

Figure 5(a) displays the scenario of epitaxially grown nanoparticles featuring an angular in-plane mosaicity distribution $\Delta \Theta$ which, as illustrated in the inset at the figure top, is well described by a Gaussian distribution. The sketched orange particles are perfectly aligned to the given substrate orientation, whereas the blue (green) particles are tilted counterclockwise (clockwise) by $+\Delta \Theta / 2(-\Delta \Theta / 2)$ with respect to the preferred direction around the substrate normal. The high-energy reciprocal-space map $(E=85 \mathrm{keV})$ included in Fig. 5(a) was measured on such a sample system with epitaxial $\mathrm{Rh}$ nanoparticles grown on $\mathrm{MgAl}_{2} \mathrm{O}_{4}(001)$ [20]. The map was probed in a single snapshot with the sample aligned to the (111) Bragg peak of the untilted (here: orange) particles. Accordingly, as illustrated in Fig. 5(a), the Ewald sphere intersects the rod of the untilted particles (vertical orange bar) at the position of the (111) Bragg peak (closed orange circle) resulting in the detection of the (111) reflection.

As can be inferred from the measured 2D map, a much wider range in reciprocal space in addition to the particle (111) Bragg reflection is detectable, and it is the particles' in-plane mosaicity that facilitates the measurability of signals over this wide $\left(\vec{q}_{\perp}, \vec{q}_{||}\right)$-range by compensating for the missing rocking scan at a fixed sample rotation $\theta_{\mathrm{S}}$. Hence, as indicated 


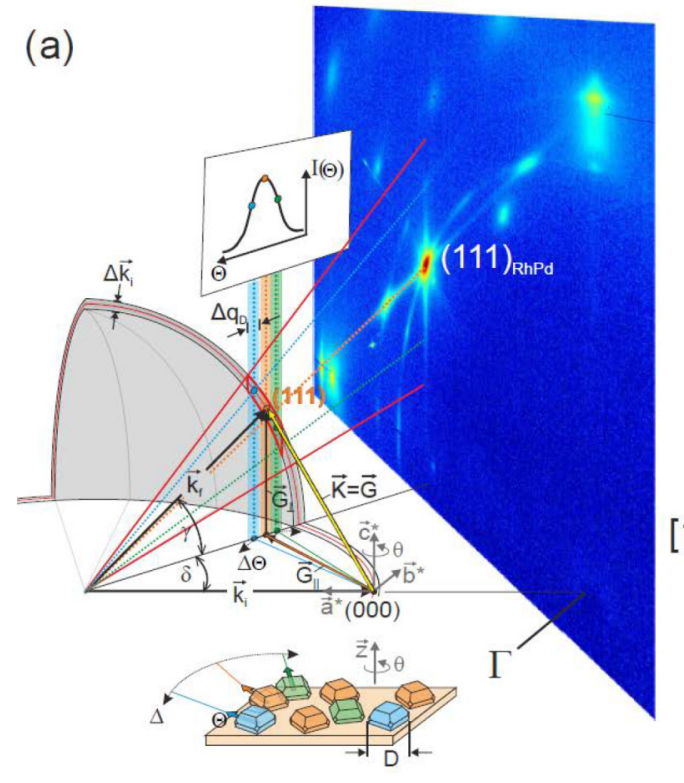

(b)
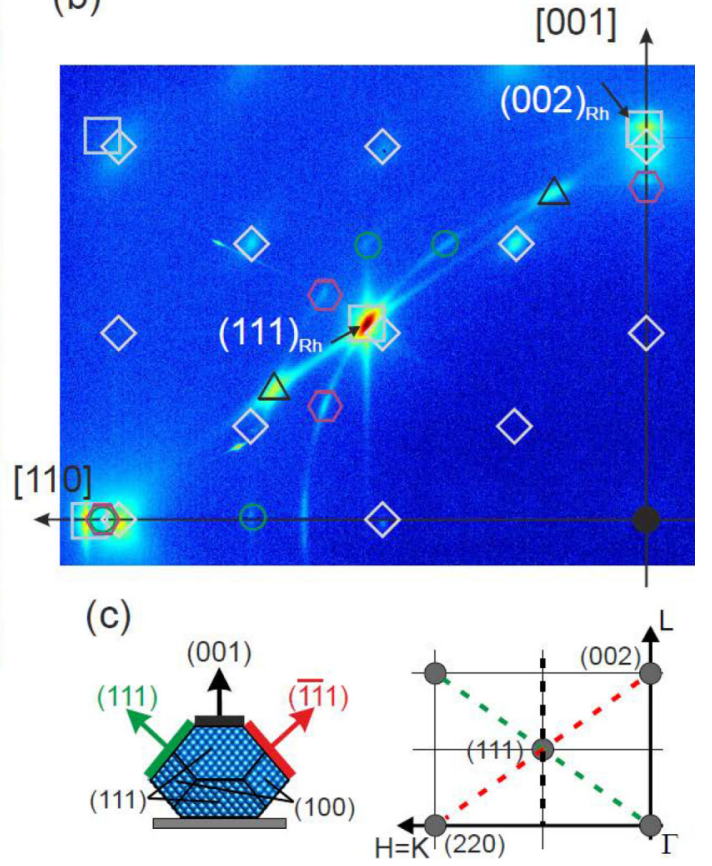

FIG. 5. High-energy diffraction measurement scheme for epitaxial nanoparticles: (a) perspective view of the diffraction geometry for nanoparticles with an in-plane angular distribution $\Delta \Theta$ and finite diameter $D$; (b) diffraction pattern from epitaxial Rh nanoparticles on $\mathrm{MgAl}_{2} \mathrm{O}_{4}(100)$ recorded by a 2D detector in stationary geometry with fixed sample rotation $\theta_{\mathrm{S}}$; (c) (left) schematic view of a (100)-oriented, truncated octahedral nanoparticle with (001) top and (111) side facets, (right) corresponding reciprocal lattice with facet rod signals indicated.

in Fig. 5(a), the rods from particles tilted counterclockwise (clockwise) around the surface normal intersect the Ewald sphere at higher (lower) out-of-plane angles. This results in intensities above (below) the (111) reflection, hence enlarging the range of detectable signals along the particle rod in the $\vec{q}_{\perp}$ direction with the intensity modulated by a Gaussian function. An additional, but less pronounced, extension of the measurable area along $\vec{q}_{\perp}$ can be traced back to the finite particle width $D$ (typically $\leqslant 10 \mathrm{~nm}$ ), leading to a broadening $\Delta q_{D}=\frac{2 \pi}{D}$ of the respective rods. The borders of the resulting overall detectable range along $\vec{q}_{\perp}$ are sketched by the red lines in Fig. 5(a). Applying this scenario to other vertical cuts through the Ewald sphere explains the additional detectability along the $\vec{q}_{\|}$direction, spanning the measureable $\left(\vec{q}_{\perp}, \vec{q}_{\|}\right)$space.

To illustrate the richness in information the accessible $\left(\vec{q}_{\perp}, q_{\|}\right)$space allows us to obtain on nanoparticle systems, Fig. 5(b) displays the reciprocal space map of Fig. 5(a) along with indications for various measured signals. Such a 2D map, probing the substrate $(H=K, L)$ plane, contains for instance quantitative information on the main particle epitaxy consisting of (001)-oriented and truncated octahedral-shaped particles [see Fig. 5(c)]: their size can be deduced from the full widths at half maximum of the indicated (111) and (002) Bragg reflections, their quantitative shape from the facet signals oriented perpendicular to the corresponding particle facets, respectively. In addition, also the presence, percentage, and size of particles belonging to other epitaxies such as (111)-oriented particles (hexagons) or (110)-oriented particles (open circles), can be inferred from the respective Bragg reflections. Moreover, the substrate reflections (diamonds) allow for a direct identification of the respective epitaxial relationships, while information on particle growth defects ("internal twinning," upward triangles) also can be deduced.

To estimate the amount of reciprocal space that can be probed in stationary geometry with $\theta=$ const. $=\theta_{\mathrm{S}}$, the parametrization in Eq. (1) or (1a) was employed in Eq. (2) or (2a) for high- and low-photon energies, respectively, to calculate $\gamma$ as a function of $\delta$, covering again the same in-and out-of-plane momentum transfer range $q_{\|}$and $q_{\perp}$ of 70 and $60 \mathrm{~nm}^{-1}$, respectively. In Fig. 6(a) and 6(b) the $\gamma(\delta)$ trajectories are plotted for photon energies of 88.6 and $12.4 \mathrm{keV}$, respectively, with $\theta_{\mathrm{S}}$ fixed at the $\operatorname{Pd}(111) \mathrm{Bragg}$ angle for a (100)-oriented surface in the $(h=k, l)$ plane. For
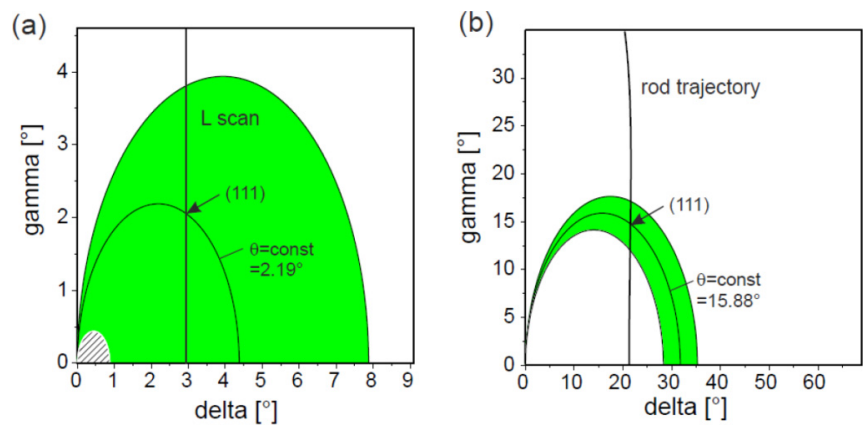

FIG. 6. (a) Accessible area in reciprocal space at high-photon energies for a nanoparticle mosaic distribution of $\Delta \Theta=3.5^{\circ}$ around the surface normal (green). (b) Accessible area of reciprocal space at $12.4 \mathrm{keV}$ is indicated (green). The half circles in the $(\delta, \gamma)$ plane fulfilling the scattering condition and going through the (111) Bragg peak are plotted as solid lines. 
both energies the trajectories constitute, in the $(\delta, \gamma(\delta))$ plane, in good approximation, semicircles centered at $\delta=\theta_{\mathrm{S}}$ and $\gamma=0^{\circ}$, with radius $\theta_{\mathrm{S}}$. For a fixed angle $\theta_{\mathrm{S}}$ reciprocal space is probed along these circles at once, even for a perfect sample.

Due to the differently tilted particles within the mosaic spread, the diffraction condition is fulfilled for a range of various in-plane diffraction angles $\theta_{\mathrm{B}}$ at a stationary sample angle $\theta_{\mathrm{S}}$. Taking an in-plane mosaicity with full width at half maximum $\Delta \Theta$ into account, we can draw semicircles for $\theta_{\mathrm{S}}-\Delta \Theta / 2$ and $\theta_{\mathrm{S}}+\Delta \Theta / 2$, describing the range within which the diffraction condition is fulfilled due to the particles' in-plane mosaicity. Hence, the green area in Fig. 6(a) is accessible at $88.6 \mathrm{keV}$ photon energy and taking $\Delta \Theta=3.5^{\circ}$ into account; for comparison the much smaller reciprocalspace area for $\Delta \Theta=3.5^{\circ}$ at $12.4 \mathrm{keV}$ photon energy is displayed in Fig. 6(b). Hence, a much wider $\left(\vec{q}_{\perp}, \vec{q}_{\|}\right)$range in reciprocal-space plane can be imaged when employing high-energy $x$ rays. In both cases a slight (nearly identical) variation in in-plane momentum transfer perpendicular to the probed reciprocal-space plane is present, which, due to the finite in-plane peak widths, can in practice be neglected. Apart from the in-plane mosaic spread discussed so far, an out-ofplane mosaic spread is often present in sample systems in addition. It makes also the region around the specular rod with $\vec{q}_{\|}=0$ available for detection, leading in the $\mathrm{Rh}$ nanoparticle case [Fig. 5(b)] to the monitoring of the out-of-plane Bragg reflections of (001)- and (111)-oriented nanoparticles. Hence, the areas in reciprocal space accessible due to both in-plane and out-of-plane mosaicity are superimposed.

For the investigation of surfaces or interfaces in stationary geometry, the high-energy approach suggests that by tuning the sample mosaicity, substrate crystal truncation rods or surface rods originating from reconstructions or incommensurate layers can be probed over a wider $l$ range. This opens interesting opportunities to follow atomic-scale structural changes in stationary geometry during time-resolved measurements without moving the sample.

\section{DISCUSSION AND CONCLUSIONS}

The approach presented here raises the question, under which circumstances the use of high-energy photons is beneficial for the investigation of systems with reduced dimensions such as surfaces, interfaces, or nanostructures. Even in the high-energy $\mathrm{x}$-ray case (70-90 keV) the Ewald sphere is not completely flat and in a stationary geometry diffraction takes only place in directions where the reciprocal lattice intersects the Ewald sphere. For perfect surfaces or perfectly aligned nanostructures this gives rise to well-defined diffraction spots on the 2D detector, as seen in Fig. 4(a) in the case of a $\mathrm{Pd}(100)$ single crystal with low mosaicity. A sample rotation is still needed to record the whole reciprocal space, which is traditionally performed at lower photon energies using a smaller 2D detector, involving a time-consuming point-topoint movement of the sample and detector arm, which can be significantly speeded up by a continuous movement of the detector arm and synchronized readout of the detector and automatized absorber system [25,26]. Alternatively, also at lower, conventional photon energies a stationary, large 2D detector could also be employed for fast data recording, together with fast online data analysis to create distortion-free cuts through reciprocal space, as they are directly delivered in very good approximation in the high-energy case. The data acquisition is still speeded up in the high-energy case, since for the detection of the useful signal in one azimuthal reciprocal-space plane only a small angular movement of a few degrees is required and straightforward data interpretation is possible without further numerical processing.

With current detector technology, there is however one drawback when using a large 2D detector (both low- and high-energy case): it lies in the need of masking the positions of bulk Bragg reflections by placing individual small absorbers on the 2D detector to avoid detector saturation or damage. This fortunately does not represent a loss in information, since the bulk Bragg reflections do not contain surface structural information. The new generation of $2 \mathrm{D}$ pixel detectors optimized for high-photon energies [27] overcomes some of these shortcomings by providing a largely enhanced dynamical range. A future high-photon-energy x-ray detector development on the basis of the principle of the adaptive gain integrating pixel detector is expected to fully overcome this technical problem [28].

The real benefit using high-energy photons lies in the investigation of systems with slight imperfections such as mosaicity on the order of the in-plane diffraction angle $\theta_{\mathrm{B}}$ or reduced domain size. In the case of a stationary geometry, in which the sample is not rotated, these imperfections make a wide range of a vertical reciprocal-space plane detectable, as was pointed out in Sec. III. This is very beneficial for time-resolved in situ and operando experiments monitoring growth, catalytic, or electrochemical processes on surfaces and nanoparticles [18]. In addition, it is also highly attractive for the investigation of ultrafast phenomena on time scales from ns to fs during pump-probe experiments. High-photon energies are naturally suited for the investigation of interfaces buried deeply underneath strongly absorbing materials [2931]. The recently proposed investigation of free surfaces in normal incidence transmission mode [32] is promising but can be expected to suffer from a worse signal-to-noise ratio, hampering quantitative data analysis.

In conclusion, we have elucidated the working principles of high-energy x-ray reciprocal-space mapping for the investigation of surfaces, interfaces, epitaxial nanoparticles, and nanostructures. We have demonstrated that, in good approximation, a distortion-free mapping of reciprocal-space planes perpendicular to the surface can be performed in stationary geometry employing a suitably large 2D detector and we have highlighted the important role of controlled defect structures in the sample for this purpose. This opens up new possibilities for operando and time-resolved experiments with atomic-scale structural resolution at third-generation synchrotron sources providing sufficient flux in the hard $\mathrm{x}$-ray regime and emphasizes the need for high-energy photons at free-electron laser x-ray sources for ultrashort-time pumpprobe experiments.

\section{ACKNOWLEDGMENTS}

The authors thank Robert Feidenhans'l and Jakub Drnec for fruitful discussions. Olof Gutowski is acknowledged for 
his technical support during the beamtimes at P07, PETRA III. Financial support by the Bundesministerium für Bildung und Forschung (BMBF), Project No. 05K10PS1 (NanoXcat) is gratefully acknowledged. This work was performed within the Röntgen-Ångström collaboration "Catalysis on the atomic scale".

\section{APPENDIX}

The use of high-photon energies makes a small-angle approximation $\left[\sin x=x, \cos x=1\right.$, valid up to $x=0.14 \operatorname{rad}\left(8^{\circ}\right)$ within $1 \%$ error] feasible, which allows a reformulation of the aforementioned scattering-angle parametrization:

$$
\gamma=\alpha^{2}+4 \theta_{\mathrm{B}}\left(\theta-\theta_{\mathrm{B}}\right) \cong 4 \theta_{\mathrm{B}}\left(\theta-\theta_{\mathrm{B}}\right)
$$

(within $1 \%$ for $\gamma \geqslant 7 \alpha$ )

$$
\begin{gathered}
\gamma \cong 2 \sqrt{\theta_{\mathrm{B}}\left(\theta-\theta_{\mathrm{B}}\right)}, \\
\delta=\frac{2 \theta_{\mathrm{B}}}{\left[1-4 \theta_{\mathrm{B}}\left(\theta-\theta_{\mathrm{B}}\right)\right]^{1 / 2}}=\frac{2 \theta_{\mathrm{B}}}{\left[1-\gamma^{2}+\alpha^{2}\right]^{1 / 2}} \cong 2 \theta_{\mathrm{B}},
\end{gathered}
$$

(within $1 \%$ for $\theta_{\mathrm{B}}>4^{\circ}$ and $\gamma<8^{\circ}$, or within $4 \%$ for $\theta_{\mathrm{B}}>1^{\circ}$ and $\gamma<8^{\circ}$ ).

Further, it follows from Eq. (3):

$$
1-\frac{1}{2}(2 \vartheta)^{2}=\left(1-\frac{1}{2} \delta^{2}\right)\left(1-\frac{1}{2} \gamma^{2}\right) \cong 1-\frac{1}{2} \delta^{2}-\frac{1}{2} \gamma^{2},
$$

and

$$
2 \vartheta \cong \sqrt{\delta^{2}+\gamma^{2}}
$$

[1] I. K. Robinson and D. J. Tweet, Rep. Prog. Phys. 55, 599 (1992).

[2] R. Feidenhans'l, Surf. Sci. Rep. 10, 105 (1989).

[3] H. Dosch, Critical Phenomena at Surfaces and Interfaces Evanescent X-Ray and Neutron Scattering, Springer Tracts in Modern Physics (Springer, New York, 1992), Vol. 126.

[4] E. Vlieg, J. Appl. Cryst. 30, 532 (1997).

[5] A. Stierle and E. Vlieg, in Modern Diffraction Methods, edited by E. J. Mittemeijer and U. Welzel (Wiley VHC, Weinheim, 2013).

[6] G. Renaud, Surf. Sci. Rep. 32, 5 (1998).

[7] T. N. Jensen, M. K. Rasmussen, J. Knudsen, A. Vlad, S. Volkov, E. Lundgren, A. Stierle, and J. V. Lauritsen, Phys. Chem. Chem. Phys. 17, 5795 (2015).

[8] A. Stierle, J. Gustafson, and E. Lundgren, in Operando Research in Heterogeneous Catalysis, Springer Series in Chemical Physics, edited by J. Frenken and I. Groot (Springer International Publishing, Switzerland, 2017), Vol. 114.

[9] R. Westerström, J. G. Wang, M. D. Ackermann, J. Gustafson, A. Resta, A. Mikkelsen, J. N. Andersen, E. Lundgren, O. Balmes, X. Torrelles, J. W. M. Frenken, and B. Hammer, J. Phys.: Condens. Matter 20, 184018 (2008).

[10] M. D. Ackermann, T. M. Pedersen, B. L. M. Hendriksen, O. Robach, S. C. Bobaru, I. Popa, C. Quirós, H. Kim, B. Hammer, S. Ferrer, and J. W. M. Frenken, Phys. Rev. Lett. 95, 255505 (2005).

[11] P. Nolte, A. Stierle, N. Y. Jin-Phillipp, N. Kasper, T. U. Schulli, and H. Dosch, Science 321, 1654 (2008).

[12] P. Nolte, A. Stierle, N. Kasper, N. Jeutter, and H. Dosch, Nano Lett. 11, 4697 (2011).

[13] U. Hejral, A. Vlad, P. Nolte, and A. Stierle, J. Phys. Chem. C 117, 19955 (2013).

[14] A. Stierle, Int. J. Mater. Res. 100, 1308 (2009).
[15] P. Nolte, A. Stierle, O. Balmes, V. Srot, P. A. van Aken, L. P. H. Jeurgens, and H. Dosch, Catal. Today 145, 243 (2009).

[16] J. Gustafson, M. Shipilin, C. Zhang, A. Stierle, U. Hejral, U. Ruett, O. Gutowski, P.-A. Carlsson, M. Skoglundh, and E. Lundgren, Science 343, 758 (2014).

[17] M. Shipilin, U. Hejral, E. Lundgren, L. R. Merte, C. Zhang, A. Stierle, U. Ruett, O. Gutowski, M. Skoglundh, P.-A. Carlsson, and J. Gustafson, Surf. Sci. 630, 229 (2014).

[18] U. Hejral, P. Müller, O. Balmes, D. Pontoni, and A. Stierle, Nat. Commun. 7, 10964 (2016).

[19] P. Nolte, A. Stierle, N. Kasper, N. Y. Jin-Phillipp, H. Reichert, A. Rühm, J. Okasinski, H. Dosch, and S. Schöder, Phys. Rev. B 77, 115444 (2008)

[20] P. Müller, U. Hejral, U. Rütt, and A. Stierle, Phys. Chem. Chem. Phys. 16, 13866 (2014).

[21] H. Reichert, V. Honkimäki, A. Snigirev, S. Engemann, and H. Dosch, Physica B 336, 46 (2003).

[22] A. Stierle, A. Steinhäuser, A. Rühm, F. U. Renner, R. Weigel, N. Kasper, and H. Dosch, Rev. Sci. Instrum. 75, 5302 (2004).

[23] C. M. Schlepütz, R. Herger, P. R. Willmott, B. D. Patterson, O. Bunk, Ch. Brönnimann, B. Henrich, G. Hülsen, and E. F. Eikenberry, Acta Crystallogr., Sect. A: Found. Adv. 61, 418 (2005).

[24] E. Vlieg, I. K. Robinson, and K. Kern, Surf. Sci. 233, 248 (1990).

[25] J. Drnec, T. Zhou, S. Pintea, W. Onderwaater, E. Vlieg, G. Renaud, and R. Felici, J. Appl. Cryst. 47, 365 (2014).

[26] A. Dawiec, Y. Garreau, J. Bisou, S. Hustache, B. Kanouté, F. Picca, G. Renaud, and A. Coati, J. Instrum. 11, P12018 (2016).

[27] https://www.dectris.com/products/pilatus3/pilatus3-x-cdte-forsynchrotron. 
[28] B. Henrich et al., Nucl. Instrum. Methods 633, S11 (2010).

[29] H. Reichert, O. Klein, H. Dosch, M. Denk, V. Honkimäki, T. Lippmann, and G. Reiter, Nature (London) 408, 839 (2000).

[30] V. Vonk, D. Pontoni, M. Cremers, A. Kerkenaar, A. A. C. Bode, W. Szweryn, G. Nowak, A. E. F. de Jong, H. Dosch, and E. Vlieg, Langmuir 33, 814 (2017).
[31] A. E. F. de Jong, V. Vonk, M. Ruat, M. Boćkowski, G. Kamler, I. Grzegory, V. Honkimäki, and E. Vlieg, J. Cryst. Growth 448, 70 (2016).

[32] F. Reikowski, T. Wiegmann, J. Stettner, J. Drnec, V. Honkimäki, F. Maroun, P. Allongue, and O. M. Magnussen, J. Phys. Chem. Lett. 8, 1067 (2017). 